\title{
Silver Nanoparticles as Enhancing Substrates for Raman and Fluorescence Spectroscopy
}

\author{
M. Cyrankiewicz*, T. Wybranowski and S. Kruszewski \\ Medical Physics Division, Biophysics Department, Collegium Medicum of Nicolaus Copernicus University, ul. \\ Jagiellońska 13, 85-067 Bydgoszcz, Poland
}

\begin{abstract}
The enhancing properties of silver nanoparticles in surface-enhanced Raman scattering (SERS) and metalenhanced fluorescence (MEF) are studied in this work. The obtained results confirm that the partial aggregation of nanoparticles leads to a great increase of Raman scattering cross-section but there are significant differences in SERS-activity of colloidal silver treated with various aggregating compounds. The differences are interpreted through the analysis of both experimental and computational results. The same silver colloid covered with silica shell preventing the fluorescence quenching makes possible a several-fold increase in fluorescence emission. The effect strongly depends on thickness of the outer layer of nanoparticles. Geometrical parameters of nanoparticles (radius or radius and thickness of the adsorption layer in core-shell systems) are determined on the basis of the dynamic light scattering (DLS) data and extinction spectra analysis.
\end{abstract}

DOI: 10.12693/APhysPolA.125.A-11

PACS: 33.20.Fb, 33.20.Kf, 32.50.+d, 61.46.Df, 73.20.Mf, 78.67.Bf, 87.83.+a

\section{Introduction}

Metallic nanoparticles (especially silver and gold) exhibit interesting optical properties related to the excitation of localized surface plasmons (LSP). Interaction of the incident light and free electrons in the conduction band results in a strong absorption (extinction) and high enhancement of the EM field in near-field region around nanoparticles. The characteristic plasmonic frequencies strongly depend on sizes and shapes of nanostructures as well as on the dielectric function of metal $[1,2]$. The near-field enhancement is profited by Raman and fluorescence spectroscopy techniques improving their detection sensitivities.

Surface-enhanced Raman scattering (SERS) is a combination of two mechanisms. The electromagnetic mechanism of SERS is related to the previously mentioned resonant LSP exitation [3]. The second contribution to the SERS enhancement is a short-range resonant mechanisms of chemical nature: chemical bonding and charge-transfer transitions between metallic nanoparticles and coordinated molecules [4]. On isolated nanoparticles, both mechanisms allow to obtain local field enhancement up to about 6 orders of magnitude but the average intensity of Raman scattering from molecules dissolved in macroscopic suspension of such nanoparticles can increase by only $2-3$ orders of magnitude in comparison with pure solution of Raman scatterers. This enhancement is far from being sufficient for practical applications. SERS signal can be further amplified through partial aggregation of colloid [5]. This approach is based on the fact that the extremely high electric field is induced in the narrow

*corresponding author; e-mail: micy@cm.umk.pl slits between nanoparticles, in the so-called "hot-spots". The intensity of Raman scattered light originated from molecules occupying such places may rise up to $10^{12}$ fold ( $10^{15}$-fold as the result of both mechanisms) [6]. It makes possible to detect SERS signal from a single molecule $[7,8]$. This paper is an attempt to interpret the observed differences in SERS-activity of silver nanoparticles treated with various aggregating compounds. Rhodamine $6 \mathrm{G}$ is used here as a SERS probe molecule. The authors analyze and compare SERS-efficiency, absorption, and scattering properties and structure of aggregates formed under the influence of chloride $(\mathrm{KCl})$ or a compound which does not contain chloride ions $\left(\mathrm{HNO}_{3}\right)$.

The second phenomenon where the unique plasmonic properties of noble metal nanostructures play a substantial role is the metal-enhanced fluorescence (MEF) [9]. The total increase in the intensity of fluorescence light is a result of two resonance processes: enhancement of excitation and enhancement of emission of fluorophores placed in the vicinity of nanoparticles [10]. The enhancement of excitation is related to the increase of the excitation rate of fluorescence molecules and is proportional to the strength of the local EM field at their location. When excited molecules are near metallic nanoparticles, the light emitted by these molecules interacts resonantly with LSP that leads to faster deactivation of fluorophores and, as a consequence, results in an increase in the quantum yield and also (in most cases) a decrease in lifetime. One should note that the adsorption of a dye directly on a metal surface results in a quenching of its fluorescence. To avoid the quenching, the nanoparticles have to be coated with a thin layer of dielectric and nonfluorescent material. For the preliminary MEF studies presented in this work, silica-coated silver nanoparticles were used. FITC molecules were used as a fluorescent probes. 


\section{Experimental}

\subsection{Materials}

Silver nitrate, trisodium citrate, potassium chloride, ethanol and ammonia $\left(\mathrm{NH}_{3}, 25 \%\right)$ were obtained from POCh S.A. (Poland). Tetraethyl-orthosilicate (TEOS) and (3-aminopropyl)triethoxysilane (APS) were obtained from Fluka (Switzerland). Rhodamine 6G was purchased from Sigma-Aldrich. Mili-Q grade water was used for preparation of all solutions.

\subsection{Silver colloids production and samples preparation}

The silver colloid used in experiments was prepared using a slightly modified Lee and Meisel method [11].

SERS experiment. The method of the preparation of both pure nanoparticles and samples for SERS studies was described in our previous paper [12]. Simultaneous measurements (recording of SERS and extinction spectra as well as particle sizing by DLS) were started immediately after the preparation of each sample.

MEF experiment. (Step 1) Before the use of the silver nanoparticles, the originally obtained suspension was purified by centrifugation at $6000 \mathrm{rpm}$ for $8 \mathrm{~min}$ and the precipitate was dissolved in ethanol to the resultant colloid concentration 2.5-fold smaller (determined by absorbance measurements) than the original mixture. Then, the ethanolic solution of silver nanoparticles was divided into 12 portions of $3.95 \mathrm{~mL}$ each. Next, $16 \mu \mathrm{L}$ of ammonia and $36 \mu \mathrm{L}$ of water was added to each of them. Finally, proper amount of TEOS (from $0.2 \mu \mathrm{L}$ to $1.0 \mu \mathrm{L}$ with a step of $0.1 \mu \mathrm{L}, 1.2 \mu \mathrm{L}$ and $1.5 \mu \mathrm{L})$ was rapidly injected and the reaction was continued on the shaker for the next 12 hours (at room temperature). As a control, one sample was left without TEOS.

(Step 2) In order to deposit FITC molecules on the nanoparticles, $1.7 \mathrm{mg}$ FITC was dissolved in $300 \mu \mathrm{L}$ of ethanol. After the addition of $5 \mu \mathrm{L}$ APS, the mixture was carefully stirred. A certain volume of the resultant solution was then 100-fold diluted in ethanol and the reactants were allowed to react in the dark for 24 hours. After this time, $50 \mu \mathrm{L}$ of this solution was added to each of $1 \mathrm{~mL} \mathrm{10-fold} \mathrm{diluted} \mathrm{with} \mathrm{ethanol} \mathrm{dispersions} \mathrm{prepared}$ in Step 1. The samples were stirred for $30 \mathrm{~min}$. After that, $10 \mu \mathrm{L}$ of $1 \%$ TEOS was injected to all test-tubes, which were subsequently placed in the dark for the next 24 hours. The final concentration of FITC was $7.5 \mu \mathrm{M}$.

\subsection{Instrumentation}

The test apparatus used for recording the absorption and Raman spectra as well as the particle sizing with DLS method [13] was described in detail in [12].

Steady-state fluorescence spectra were obtained on QuantaMaster (Photon Technology International, Birmingham, NJ, USA) spectrofluorometer with some homemade modifications. For fluorescence excitation of FITC, a monochromatized light from tungsten lamp (480 nm) was applied.
Both SERS and MEF experiments as well as the procedure of determination of geometrical parameters of silicacoated nanoparticles consisted of a single series of measurements (recordings of extinction, Raman and fluorescence spectra, and DLS measurements, respectively).

\section{Numerical algorithm for the size (shape) distribution retrieval}

According to Lambert-Beer's law, the absorbance of non-interacting spherical particles suspension can be described as

$$
E \approx E_{\mathrm{LB}}=\int_{r_{\min }}^{r_{\max }} C_{\text {ext }}(\lambda, r) f(r) \mathrm{d} r,
$$

where $C_{\text {ext }}$ denotes the extinction cross-section of particle of radius $r$. The relation between noiseless absorbance $E_{\mathrm{LB}}$ (the measured absorbance Einevitably includes some error) and the unknown size distribution function $f$ ( $C_{\text {ext }}$ is a weighted (kernel) function that characterizes this relationship) is a Fredholm integral equation of the first kind. The above formula can be easily extended to the two- or more-dimensional problem (e.g. a suspension of layered particles characterized by core radius $r$ and shell thickness $t$, see Sect. 4.2.). Finding the unknown size (but also shape or other features of the studied system) distribution function $f$ for a known kernel and right-hand side belongs to the class of illposed problems that can be successfully solved applying Tikhonov's [14] regularization technique. Denoting by $\boldsymbol{C}$ the matrix containing calculated spectra (as columns), and by $\boldsymbol{e}$ the column vector representing experimental data, the regularization problem can be defined as

$$
\min _{\boldsymbol{f}}\left(\|\boldsymbol{C} \boldsymbol{f}-\boldsymbol{e}\|^{2}+\alpha^{2}\|\boldsymbol{L} \boldsymbol{f}\|^{2}\right)
$$

where $\alpha$ is called the regularization parameter and wgL is an operator chosen to obtain a solution with desirable properties (e.g. identity matrix). According to the definition of the Tikhonov's functional (expression (2)), the regularized solution $f$ (column vector) can be written as

$$
\boldsymbol{f}_{\alpha}=\underset{\boldsymbol{f}}{\arg \min }\left\|\left[\begin{array}{c}
\boldsymbol{C} \\
\alpha \boldsymbol{L}
\end{array}\right] \boldsymbol{f}-\left[\begin{array}{l}
\boldsymbol{e} \\
0
\end{array}\right]\right\| .
$$

In this work the solution $f$ (a nonnegative distribution function) was calculated using the NNLS algorithm [15].

The presented method not only needs a properly defined kernel function (i.e. an adequate model of the absorbance with carefully chosen dielectric permittivities of the particle materials as well as appropriate optical constants of the surrounding medium) but also requires an effective criterion for selecting the best solution with little or no a priori knowledge. The procedure gives an infinite set of solutions but it turns out that for each value of $\alpha$, exactly one solution exists. A very convenient and intuitive tool for a choice of the optimal value of $\alpha$ is the so-called $\boldsymbol{L}$-curve criterion. This name refers to the shape of parametric curve $\left(\rho_{\alpha}, \sigma_{\alpha}\right)$, where $\rho_{\alpha}=\log \left\|\boldsymbol{C} \boldsymbol{f}_{\alpha}-\boldsymbol{e}\right\|$ and $\sigma_{\alpha}=\log \left\|\boldsymbol{L} \boldsymbol{f}_{\alpha}\right\|$. 


\section{Results and discussion}

\subsection{SERS-activity of aggregated silver nanoparticles}

It is well known that the partial aggregation of metallic nanoparticles is prerequisite for effective enhancement of Raman scattering. As one can see from Fig. 1 the result strongly depends on the amount of added aggregating compound. One can also notice significant differences between the effects of $\mathrm{KCl}$ and $\mathrm{HNO}_{3}$. The apparent differences may be also related (but in a very limited way) to the different age of colloid when it was used for both tests. Measurements with the use of $\mathrm{KCl}$ were carried out shortly after preparation of nanoparticles while the impact of $\mathrm{HNO}_{3}$ was tested about a week later.
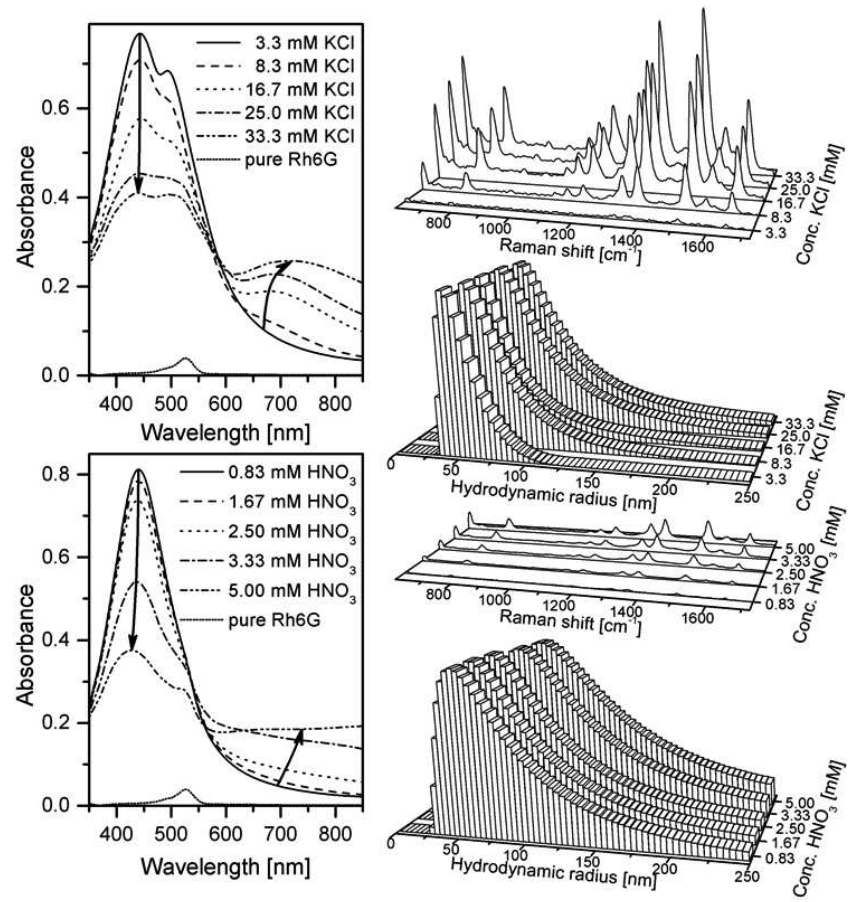

Fig. 1. Extinction and SERS spectra and the corresponding results of DLS measurements of $0.8 \mu \mathrm{M} \mathrm{Rh6G}$ on silver colloid with various amounts of $\mathrm{KCl}$ or $\mathrm{HNO}_{3}$ added to solution. Vertical scales of both Raman spectra panels are identical. The DLS intensity distribution functions are normalized to equal maximum.

The application of both aggregating agents led to the decreasing and broadening of the violet-blue absorption (extinction) band but in the case of chloride, a second spectral band in the red region arose and then increased and shifted towards longer wavelengths with the increasing concentration of $\mathrm{KCl}$.

After addition of both compounds, the maximum of DLS intensity distribution function shifted to the larger values of hydrodynamic radii but the results of DLS measurements of samples acidified with $\mathrm{HNO}_{3}$ indicated much larger changes (broadening) of the particle (or aggregate) sizes spectrum. It was consistent with a low stability of the samples.
TABLE

The composition of silver colloids calculated on the basis of analysis of the experimental spectra.

\begin{tabular}{|c|c|c|c|c|c|c|c|c|}
\hline \multirow[t]{2}{*}{ Code } & \multirow[t]{2}{*}{ Struct } & \multirow[t]{2}{*}{ NPs } & \multicolumn{3}{|c|}{ Colloid with $\mathrm{KCl}$} & \multicolumn{3}{|c|}{ Colloid with $\mathrm{HNO}_{3}$} \\
\hline & & & $\mathrm{R}[\mathrm{nm}]$ & $\mathrm{D}[\mathrm{nm}]$ & $\%$ & $\mathrm{R}[\mathrm{nm}]$ & $\mathrm{D}[\mathrm{nm}]$ & $\%$ \\
\hline M & $\bullet$ & 1 & 44.55 & 1.00 & 15.7 & 37.61 & 1.00 & 12.3 \\
\hline D & & 2 & 44.95 & 1.20 & 9.8 & 37.53 & 1.04 & 9.8 \\
\hline C3 & & 3 & 44.10 & 1.38 & 7.8 & 38.03 & 1.16 & 7.4 \\
\hline L3 & & 3 & 46.23 & 1.39 & 3.6 & 35.98 & 1.04 & $\underline{8.5}$ \\
\hline $\mathrm{C} 4$ & & 4 & 41.47 & 1.50 & 5.7 & 38.78 & 1.32 & 6.0 \\
\hline L4 & & 4 & 47.53 & 1.00 & 4.2 & 36.25 & 1.02 & $\underline{9.2}$ \\
\hline C5 & & 5 & 36.70 & 1.96 & 3.4 & 39.35 & 1.36 & 4.2 \\
\hline $\mathrm{C} 7$ & & 7 & 43.14 & 1.36 & 13.3 & 36.30 & 1.10 & 9.2 \\
\hline $\mathrm{C} 8$ & & 8 & 38.34 & 1.34 & 12.8 & 40.40 & 1.32 & 1.8 \\
\hline CL9 & 8 & 9 & 41.95 & 1.35 & 3.7 & 42.90 & 1.29 & 4.9 \\
\hline C13 & & 13 & 39.49 & 1.10 & 14.1 & 48.13 & 1.50 & 1.8 \\
\hline CL13 & 888 & 13 & 42.19 & 1.35 & 2.9 & 43.20 & 1.22 & $\underline{6.4}$ \\
\hline S13 & & 13 & 44.20 & 0.80 & 3.0 & 36.64 & 0.94 & $\mathbf{1 8 . 6}$ \\
\hline & & Avg & 42.42 & 1.29 & & 38.06 & 1.11 & \\
\hline
\end{tabular}

Despite the higher degree and rate of aggregation, a relatively weak Raman signal was obtained from nanoparticles treated with $\mathrm{HNO}_{3}$. The samples containing $8 \mathrm{mM}$ and more $\mathrm{KCl}$ were significantly more SERSactive. Chloride ions can facilitate the adsorption of rhodamine $6 \mathrm{G}$ on the silver surface but in this work it is assumed that the major factor of the increase of Raman scattering intensity was the formation of assemblies that were more favorable in terms of SERS phenomena.

The authors do not have any instrumental method that allows to determine the structure of colloid at the single aggregate level. On the other hand, the sample preparation (e.g., for imaging by electron microscopy) itself can easily change the configuration of nanoparticles. The method described in Sect. 3 allows to solve (at least partially) the stated problem in a completely non-destructive way. In this work, the extinction spectra of the samples were decomposed into a set of spectra calculated for chosen regular structures. The GMM [16] computations were done for various radii of nanoparticles $(30 \mathrm{~nm}, 35 \mathrm{~nm}$, $40 \mathrm{~nm}, 45 \mathrm{~nm}$, and $50 \mathrm{~nm}$ ) and for various interparticle distances $(0.25 \mathrm{~nm}, 0.5 \mathrm{~nm}, 0.75 \mathrm{~nm}, 1 \mathrm{~nm}, 1.5 \mathrm{~nm}$, $2 \mathrm{~nm}, 3 \mathrm{~nm}$, and $5 \mathrm{~nm}$ ). The values of mean radii $(\mathrm{R})$ and mean distances (D) between nanoparticles forming the aggregates of given shapes as well as their fraction in the total number of aggregates calculated for samples with $33.3 \mathrm{mM} \mathrm{KCl}$ and $5 \mathrm{mM} \mathrm{HNO}_{3}$ are presented in Table I. The mean absolute errors (MAE) of the model 
spectra (the results of the multiplication of kernel matrices $C$ by appropriate distribution functions - column vectors $\boldsymbol{f}$, see Sect. 3) of colloids with $\mathrm{KCl}$ and $\mathrm{HNO}_{3}$ were 0.0052 (1.29\% of the maximum of absorbance of a measured spectrum) and 0.0044 (1.20\%), respectively.

Looking at Table I one can conclude that the aggregation of silver nanoparticles in two of the analyzed samples proceeded in a slightly different manner. After the addition of chloride nanoparticles tended to aggregate into more compact (cluster-like, e.g. C8 and C13) structures. Moreover, at the moment of registration of the spectrum, a relatively large part of nanoparticles remained unaggregated. The same colloid in presence of $\mathrm{HNO}_{3}$ seems to prefer the other geometric patterns of aggregation (linear-chain, L3 and L4, mixed, e.g. CL13, and star-like - S13). The obtained results should be carefully related to real colloids, however the slightly greater interparticle distance in the case of using $\mathrm{KCl}$ may indicate that the molecules of rhodamine formed a dense adsorption layer preventing direct contact between metallic particles.

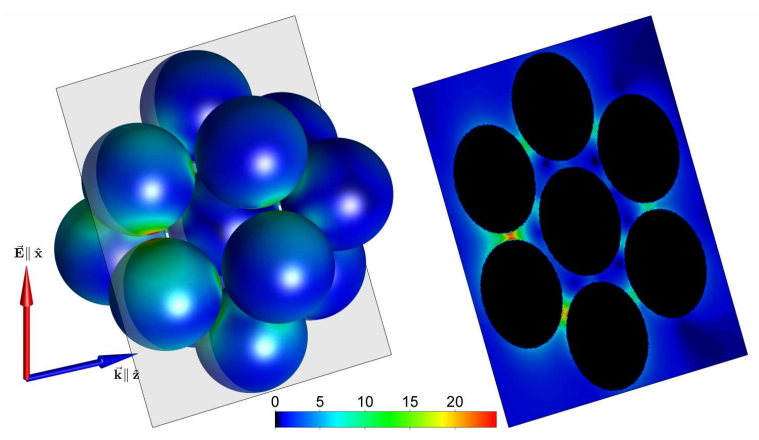

Fig. 2. Local electric field distribution around a densely packed cluster consisting of 13 spherical silver nanoparticles of radius $40 \mathrm{~nm}$ placed in water. The distance between nanospheres used in calculations is $5 \mathrm{~nm}$. The wavelength of incident light is $420 \mathrm{~nm}$.

Because the intensity of SERS provided by chlorideactivated colloids was at least 10-fold higher, it can be supposed that in these aggregates the number and width of interparticles junctions were more advantageous for SERS (as in Fig. 2). In the presented work, the maximal enhancement factor exceeded 6 orders of magnitude.

\subsection{The enhancement of fluorescence emission on silica coated silver nanoparticles}

As it was shown so far, the citrate-reduced silver colloid was capable to effectively enhance Raman signal. Before the use of these nanoparticles as a MEF substrate, they had to be coated with a dielectric layer of an appropriate thickness. This was done following the recipe given in Sect. 2.2. (Step 1). Colloids obtained at this stage were then 10-fold diluted with water. In order to determine their geometrical parameters, the extinction spectra were recorded and DLS measurements were performed as well. The main feature of the spectra (not presented here) was a wide band related to the excitation of surface plasmons localized on silver nanoparticles. The absorbance in this region was about 0.3 . LSPR band shifted toward longer wavelengths as the amount of added silica precursor (TEOS) increased. The collected extinction spectra were analyzed using the method described in Sect. 3. The kernel matrix $C$ was created from the spectra (250$700 \mathrm{~nm}$ ) calculated on the basis of the extension of Mie theory given by Aden and Kerker [17]. Both the radii of silver cores and thicknesses of the outer silica shells were computed. MAE of numerically reproduced spectra ranged between 0.0029 and 0.0060 (1-2\%). Fig. 3 (left graph) shows that the results obtained from both method (regularization applied to the extinction spectra and analysis of DLS) are consistent. The mean outer radii of all colloids studied here are almost identical in both cases.
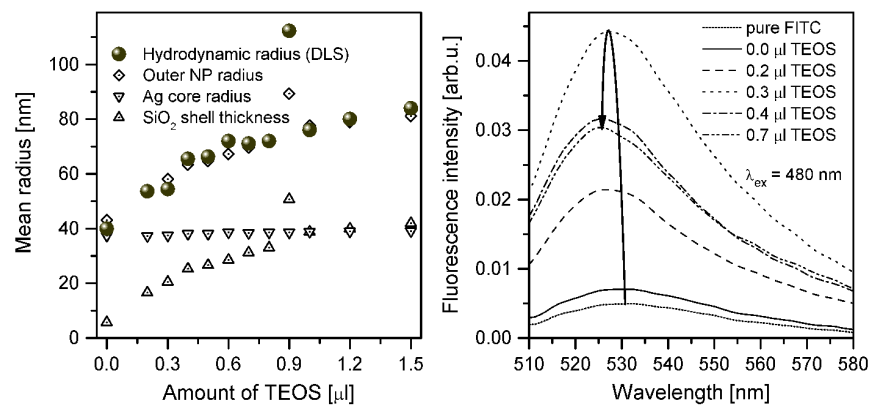

Fig. 3. Geometrical parameters of silica-coated silver nanoparticles obtained by DLS and extinction spectra analysis for colloids prepared with various amount of TEOS (left graph) and the corresponding fluorescence spectra of FITC adsorbed on these colloids (right graph).

The colloids analyzed above were then used in the proper MEF experiment. Unfortunately, some samples were destroyed in the course of the procedure described in Sect. 2.2 (Step 2). The remaining ones were placed in sequence in a chamber of the spectrofluorimeter and excited at the wavelength of $480 \mathrm{~nm}$. The recorded fluorescence emission spectra are shown on the right graph of Fig. 3. As one can see, the strongest signal was obtained for the nanoparticles prepared with $0.3 \mu \mathrm{L}$ TEOS (in Step 1). Such amount of the compound corresponds to the silica layer with an average thickness of about 20 nm. The obtained result is consistent with the observations reported in $[18,19]$. Only a mild enhancement of the fluorescence emission of FITC (approximately 9-fold increase relative to pure $7.5 \mu \mathrm{M}$ FITC solution) obtained in a single attempt does not allow to draw any firm conclusions. More detailed studies are needed to reveal the nature of the observed gain. Also the method of coating the nanoparticles with silica and depositing on them chosen fluorescent molecules requires further refinement. 


\section{Conclusions}

Agglomeration of nanoparticles (and, as a consequence, creation of narrow slits between them with the so-called "hot spots") was found to be a prerequisite for efficient SERS. In this work, the nitric acid, probably due to its acidic nature, caused much stronger aggregation than $\mathrm{KCl}$. However, the observed Raman signal was significantly weaker in this case. The higher SERS enhancement factor observed in samples treated with $\mathrm{KCl}$ was the result of both the co-adsorption rhodamine $6 \mathrm{G}$ and chloride ions on the surface of metal as well as the formation of nanoparticle assemblies exhibiting optical properties more preferred from the point of view of SERS. An essential part of the work has been devoted to the analysis of structures of these aggregates (and also their effect on the extinction spectrum and SERS-efficiency of colloid). Within the analysis, the results of experimental and computational studies were successfully confronted with each other. The both approaches lead to the conclusion that nanoparticles in presence of $\mathrm{KCl}$ tended to aggregate into more compact cluster-like structures while the same colloid with the addition of $\mathrm{HNO}_{3}$ arranged into more loose and extended structures.

The same colloid (in non-aggregated form) coated with silica layer preventing the fluorescence quenching allowed to observe a several-fold enhancement of fluorescence emission of FITC. The highest fluorescence intensity was obtained for silica shell about $20 \mathrm{~nm}$ thick.

The geometrical parameters of nanoparticles (radii or radii and thicknesses of the outer layer in core-shell systems) were determined on the basis of analysis of absorption spectra. The results were consistent with those obtained by traditional DLS methods.

\section{References}

[1] A. Derkachova, K. Kolwas, Eur. J. Phys. Special Topics 144, 93 (2007).

[2] A.L. Gonzalez, C. Noguez, Phys. Stat. Sol. (c) 4, 4118 (2007)
[3] M. Moskovits, J. Raman Spectrosc. 36, 485 (2005).

[4] P. Hildebrandt, M. Stockburger, J. Phys. Chem. 88, 5935 (1984).

[5] H. Bengter, C. Tengroth, S.P. Jacobsson, J. Raman Spectrosc. 36, 1015 (2005).

[6] S. Kruszewski, M Cyrankiewicz, Acta Phys. Pol. A 121, A-68 (2012).

[7] K. Kneipp, Y. Wang, H. Kneipp, L.T. Perelman, I. Itzkan, R.R. Dasari, M.S. Feld, Phys. Rev. Lett. 78, 1667 (1997).

[8] S. Nie, S.R. Emory, Science 275, 1102 (1997).

[9] J.R. Lakowicz, Anal. Biochem. 337, 171 (2005).

[10] S. Kruszewski, T. Wybranowski, M. Cyrankiewicz, B. Ziomkowska, A. Pawlaczyk, Acta Phys. Pol. A 113, 1599 (2008).

[11] P. C. Lee, D. Meisel, J. Phys. Chem. 86, 3391 (1982).

[12] S. Kruszewski, M. Cyrankiewicz, Acta Phys. Pol. A 119, 1018 (2011).

[13] R. Pecora, Dynamic Light Scattering: Applications of Photon Correlation Spectroscopy, Plenum Press, New York 1985.

[14] A.N. Tikhonov, V.Y. Arsenin, Solutions of Ill-posed Problems, V.H. Winston \& Sons, Washington 1977.

[15] C.L. Lawson, R.J. Hanson, Solving Least Squares Problems, Prentice-Hall, Englewood Cliffs (NJ) 1974.

[16] Y.-L. Xu, B.A.S. Gustafson, Recent Res. Devel. Optics 3, 599 (2003).

[17] A.L. Aden, M. Kerker, J. Appl. Phys. 22, 1242 (1951).

[18] O.G. Tovmachenko, C. Graf, D.J. van den Heuvel, A. van Blaaderen, H.C. Gerritsen, Adv. Mater. 18, 91 (2006).

[19] D. Cheng, Q.H. Xu, Chem. Commun. 2007, (3) 248 (2007). 